PRePARED For SUbMission to JINST

\title{
PymePix: A python library for SPIDR readout of Timepix3
}

\author{
Ahmed F. Al-Refaie, ${ }^{a}$ Melby Johny, ${ }^{a, b}$ Jonathan Correa, ${ }^{c}$ David Pennicard, ${ }^{c}$ Peter Svihra, ${ }^{d, e}$ \\ Andrei Nomerotski, ${ }^{f}$ Sebastian Trippel, ${ }^{a, g}$ and Jochen Küpper ${ }^{a, b, g}$ \\ ${ }^{a}$ Center for Free-Electron Laser Science, Deutsches Elektronen-Synchrotron DESY, Notkestrasse 85, 22607 Hamburg, \\ Germany \\ ${ }^{b}$ Department of Physics, Universität Hamburg, Luruper Chaussee 149, 22761 Hamburg, Germany \\ ${ }^{c}$ Deutsches Elektronen-Synchrotron DESY, Notkestrasse 85, 22607 Hamburg, Germany \\ ${ }^{d}$ Department of Physics, Faculty of Nuclear Sciences and Physical Engineering, Czech Technical University, Prague 115 19, \\ Czech Republic \\ ${ }^{e}$ School of Physics and Astronomy, University of Manchester, Manchester M139PL, United Kingdom \\ ${ }^{f}$ Physics Department, Brookhaven National Laboratory, Upton, NY 11973, USA \\ ${ }^{g}$ Center for Ultrafast Imaging, Universität Hamburg, Luruper Chaussee 149, 22761 Hamburg, Germany \\ E-mail: jochen.kuepper@cfel.de
}

Abstract: PymePix is a new Python 3 library that provides control and acquisition for the Timepix3-SPIDR hardware. The rich set of data-structures and intuitive routines reduces time and coding effort to quickly configure, acquire, and visualize data from Timepix 3 . The highly extensible high-performance data-pipeline allows for alteration of the Timepix 3 datastream into a form that is convinient for the user. This library is intended to be easily inserted into a standard scientific software stack as well as to allow for more direct interaction of Timepix 3 with interactive flavors of Python. Included with the library are two example programs using PymeP ix: pymepix-acq is a command line control and acquisition program that can capture UDP packets and decode them into pixels and triggers. The second is pymepixviewer, an online control and data-acquisition program for general use, but with features geared toward mass-spectroscopy and ion imaging. 


\section{Contents}

1 Introduction 1

2 PymePix library $\quad 2$

2.1 Data model
2.2 Connection and Acauisition 2

2.2 Connection and Acquisition 3

2.3 Configuring Timepix 4

2.4 Data processing pipeline $\quad 6$

$\begin{array}{lll}2.5 & \text { Timewalk Correction } & 8\end{array}$

3 Enduser utility programs $\quad \mathbf{8}$

3.1 Command-line data acquisition with pymepix-acq 8

3.2 Graphical user interface pymepixviewer 9

4 Conclusion $\quad 10$

\section{Introduction}

Timepix 3 is an application specific integrated circuit (ASIC) hybrid pixel detector developed by the MediPix3 collaboration [1] as the successor of previous versions of Timepix [2]. Each pixel in the $256 \times 256$ matrix acts independently and is capable of recording hits with timing and energy information at $\mathrm{MHz}$ rates. Timepix 3 can operate in either frame-based mode like standard CMOS and CCDs or a sparse data-driven mode where each pixel hit is immediately sent out as a data packet - containing information on the pixel coordinates, time of the hit, and energy deposited, with a deadtime of about $1 \mu$ s [3]. Each pixel has a customizable threshold level that determines when a hit is registered. If a signal causes a crossing of this threshold then the hit is registered along with the time-of-arrival (ToA) information and time taken for the signal to fall below the threshold, also referred to as the time-over-threshold (ToT) duration. ToA of a pixel is encoded as a 14-bit value operating at $40 \mathrm{MHz}$ giving a temporal resolution of $25 \mathrm{~ns}$ and maximum time of $83 \mu$ s. The resolution can be refined further using the 4-bit fast time of arrival (FToA) operating at $640 \mathrm{MHz}$ improving the resolution to $1.56 \mathrm{~ns}$. The ToT estimates the energy deposited into the pixel recorded as a 10-bit value at $40 \mathrm{MHz}$ giving a resolution of $25 \mathrm{~ns}$.

Reading out from Timepix3 is facilitated by an FPGA that acts as a middleman between the Timepix3 ASIC and the acquisition computer. The "Speedy PIxel Detector Readout" (SPIDR) readout system [4, 5] provides both a $10 \mathrm{Gbps}$ optical and $1 \mathrm{Gbps}$ ethernet interface with the former allowing for the full $40 \mathrm{Mhits} / \mathrm{cm}^{2} / \mathrm{s} \mathrm{hit} \mathrm{rate.}$ It also extends the ToA timestamp range to $26 \mathrm{~s}$ by including an additional 16-bit timestamp at the end of a pixel data packet. Both the ToA and SPIDR are synchronized with a global 48-bit clock. This clock can be used to further extend the timestamp range to 81 days. SPIDR also includes an external trigger input that introduces an additional trigger timestamp with a resolution of $260 \mathrm{ps}$ allowing for, e. g., event selection or time-of-flight (ToF) mass spectroscopy (MS) with a precise time reference from, for instance, a pulsed laser system. With SPIDR, 'slow' communication such as configuring Timepix and uploading pixel parameters are done through the TCP whilst 'fast' communication, such as pixel and time data packets during acquisition, is handled using UDP [4]. 
Python is rapidly growing to become the defacto language in scientific applications with its rich ecosystem of stable open-source libraries and tools that provide fast and easy data processing. Image analysis is supported by libraries such as OpenCV [6] and scipy [7] and plotting through matplotlib [8] either statically or interactively, e. g., using IPython [9], means that traditional cameras can go from hookup to acquisition to visualization to analysis with little effort and code.

For Timepix 3 , the data pipeline is far more complex. Traditionally, Timepix 3 must be configured for acquisition using a separate program such as SoPhy [10] or using the original SPIDR C++ API from NIKHEF [5] and the UDP packet-stream must then be captured to a file. Then, a decoding script must be written - or used from someone else - to correctly decode the packets. These decoded packets then require additional processing to improve time resolution. If event selection or time referencing is required triggers must be correlated to the correct pixel packets. Essentially a great deal of effort is required by the user to even begin visually inspecting and analysing the data-stream. Here, we provide the PymePix framework to bring the same ease and usability as for traditional cameras to the use of Timepix 3 with SPIDR to the scientific community.

\section{PymePix library}

PymePix is intended to bridge the gap between Timepix 3 and Python. The goal of the library is to allow a user without deep technical knowledge of Timepix 3 to establish a connection, start acquisition, and retrieve and plot pixel and timing information in as few lines of code as possible; at the same time it provides all details to unleash the full power of Timepix3-SPIDR hardware. This is achieved by classes that act as a black-box, handling all of the low level TCP communication and decoding of the UDP data-stream, presenting them in a pythonic fashion. More advanced and lower-level control of SPIDR and Timepix 3 is still available from these black-box classes or can be established directly by the user. For easy installation, it only depends on the standard python library, numpy, and scikit-learn.

One of the key features in PymePix is a high-performance data-pipeline. The currently available pipeline is capable of decoding all UDP packets, extending the timestamp and, if needed, correlating triggers to pixels at a data-rate of 1 Gbps. A user can deal with the datastream immediately, but PymePix also offers a more convenient method that can be leveraged with more interactive flavors of python such as IPython or Jupyter Notebook.

In many applications, e. g., the electron and ion imaging performed in our experiments, single "physics events" are detected by many detector pixels simultaneously. Thus, the individual-pixel information should be merged to provide the most precise information on the actual physical event. Rather than relegating this step to post-data acquisition, it instead motivated our need and inclusion of an easily customizable pipeline that any user can leverage to include their own post-processing routine, such as, e. g., centroiding or time-to-mass conversion in ion imaging, providing a more meaningful datastream directly from 'Timepix3' during data acqusition.

Whilst originally developed to simplify data-retrieval in ion and electron imaging experiments within the Controlled Molecule Imaging group [11, 12], we envision that the library will help scientific and industrial use of Timepix 3 and allow for more novel applications of the hardware. While we describe a slice of the capabilities provided by the library in this introductory article, this is not comprehensive. Full documentation is available through sphinx documentation built-in to the library code itself.

\subsection{Data model}

Before looking at how the library is used, it is useful to describe how data from Timepix 3 is represented. In PymePix, all data that is retrieved from Timepix 3 is packaged in the form of a python tuple called a message that includes a header and the actual data. The header is essentially an integer that identifies what kind of data 


\begin{tabular}{lll}
\hline \hline MessageType Identifier & Description & Data \\
\hline RawData & Raw UDP packets & packets, timestamp \\
PixelData & Decoded pixels & $x, y$, global ToA, ToT \\
TriggerData & Decoded triggers & trigger \#, global trigger Time \\
EventData & Correlated triggers & trigger \#, $x, y$, rToA, ToT \\
CentroidData & Centroided events & trigger\#, $x, y$, rToA, ToT \\
\hline \hline
\end{tabular}

Table 1. A summary of messages provided by PymePix. All items under Data are in the form of arrays except for timestamp, which is an integer. The global prefix refers to timestamps that have been extended past their original range. $r T o A$ refers to the time of arrival computed relative to the associated trigger time. All time of arrival and trigger times are in seconds. See text for further details.

the message contains. PymePix aliases these headers using the IntEnum MessageType. Here's an example message with the header MessageType.PixelData:

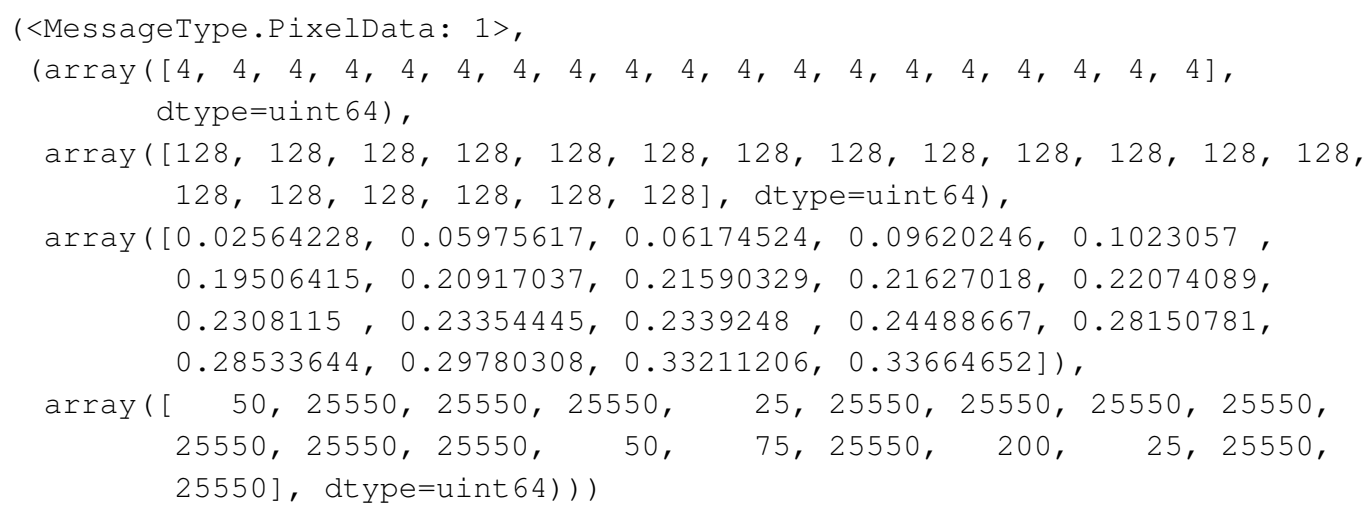

Unpacking this gives us the header and the data. The data part can be further unpacked into four separate arrays: pixel $x$ coordinate, pixel $y$ coordinate, extended pixel ToA in seconds, and ToT in seconds. For messages with arrays, the elements of each array represent the indices of a signal pixel. In our example, the first pixel was located at $(4,128)$ and arrived at $\sim 0.0256 \mathrm{~s}$. Table 1 gives a summary of the available messages, their identifiers, and data format.

\subsection{Connection and Acquisition}

PymePix provides the high-level class Pymepix that handles the connection to SPIDR, enumerates all available Timepix devices and manages acquisition. It simply requires the TCP IP address and port to SPIDR for instantiation:

>>> from pymepix import Pymepix
>> ppx = Pymepix (('192.168.1.10',50000))

Pymepix overwrites the __len__ operator so calling len gives the total number of Timepix devices connected:

|>>> len (ppx)

Acquisition can simply be started using the start method:

| >>> ppx.start () 
Acquiring data comes in two flavors: polling and callbacks. Polling is a more relaxed way of retrieving data and is the default method of acquisition. When activated, all data is placed into an internal fixed length ring buffer array. Getting data is simply a case of calling the poll method:

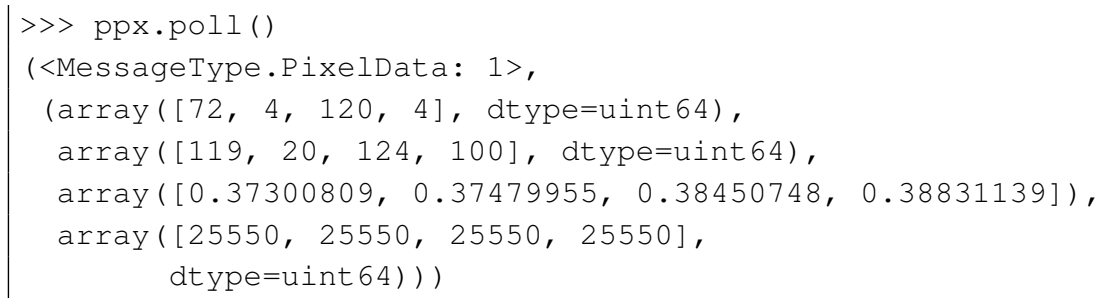

This pops the oldest value in the buffer. When new data comes in and the buffer is full, the oldest value is overwritten. An empty buffer raises a PollBufferEmpty exception.

For a more immediate handling of data, callbacks can be used. In this mode as soon as a message arrives it is split into its header and data parts and passed into the function provided by the user. This mode is immediately enabled when a function is assigned to the dataCallback property:

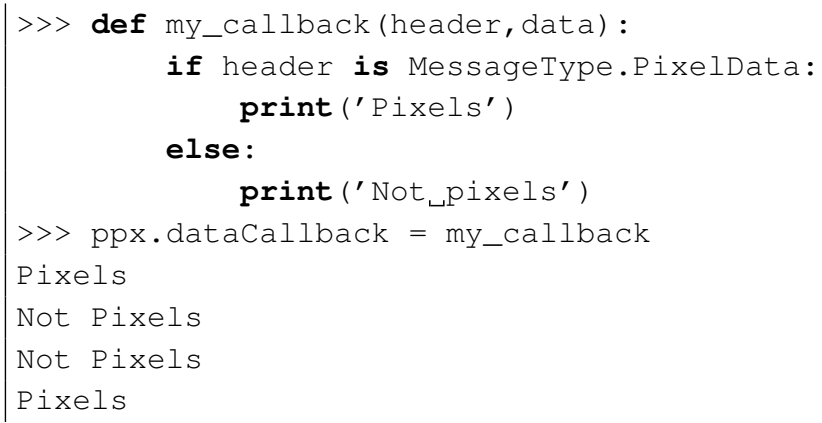

Polling is disabled at this point but can be enabled by calling the enablePolling method. Either methods can be switched between during acquisition. Of course, acquisition can be stopped through the stop method:

| >> ppx.stop ()

\subsection{Configuring Timepix}

During initialization, Pymepix enumerates connected Timepix3 devices and creates a TimepixDevice object for each one. These can be used to configure each individual Timepix 3 device and can be accessed by the square-brackets operator:

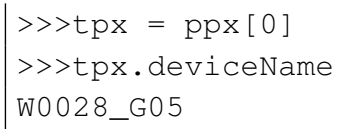

With the devicename property returning the assigned name of the Timepix 3 device. TimepixDevice gives full access to all Timepix registers and digital-to-analog-converter (DAC) parameters, such as current preamplifier settings and voltage thresholds, in the form of class properties. When dealing with registers, PymePix provides a set of enums that can be used to easily understand and set them. For instance, reading the operation mode register and switching from ToA only mode to ToA-and-ToT mode is easily and clearly achieved:

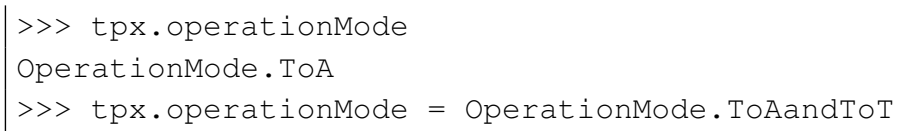


|>> tpx.operationMode

operationMode. ToAandToT

With regards to DAC parameters, it is important for previous users of Timepix3 to note that in PymePix they are now interpreted in SI units, e. g., Volt (V) and Ampere (A), rather than integer codes. Commonly tuned DAC parameters are the coarse and fine voltage thresholds. Reading and then setting these to $580 \mathrm{mV}$ and $100 \mathrm{mV}$, respectively, is achieved through

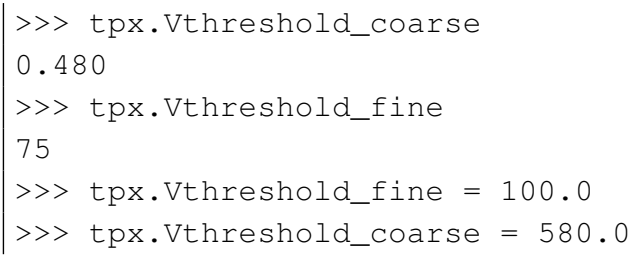

Of course if you're unsure of the units used you can always check the docstring:

nA
nA

For backward compatibility, the option to set DAC parameters using a supplied integer code and value is available through the setDac method:

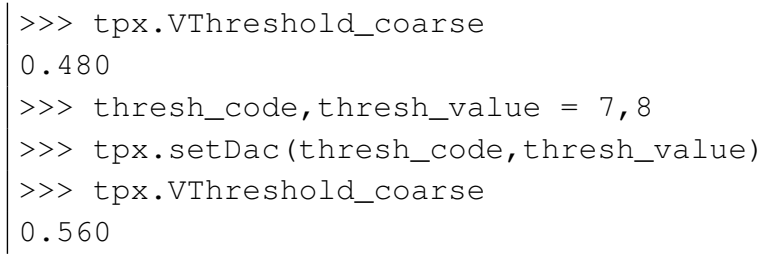

Pixel configuration is handled with the properties pixelMask for a pixel mask and pixelThreshold for the pixel DAC thresholds. They are local configurations that can be passed to and from Timepix 3 using the uploadPixels and refreshPixels methods, respectively. These are exposed as Numpy ndarrays of type numpy . uint 8 and can thus be used like any other Numpy array:

>> tpx.pixelMask $[\ldots]=1$
>> tpx.pixelThreshold $[:: 2,1:: 2]=8$

This includes being used as arguments whereever Numpy arrays are accepted

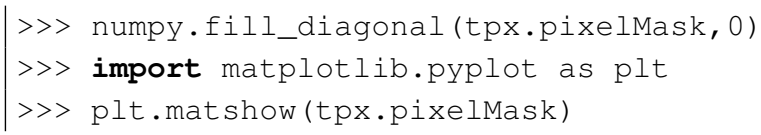

and new arrays can be assigned to them as long as they are of shape $256 \times 256$ with type numpy $\cdot$ uint 8 :

| >> tpx.pixelThreshold $=$ np.ones ( $\operatorname{shape}=(256,256)$, dtype=np.uint 8)

Assigning an array with an incorrect shape and type results in a BadP ixelFormat exception. TimepixDevice also handles loading settings and the pixel matrix from configuration files using the loadConfig method. The behaviour of loadConfig is determined by what TimepixConfig class is assigned. This class simply provides methods dacCodes, maskConfig, and thresholdConfig, which provide a list of DAC code-value pairs, the pixel mask array, and the pixel threshold array, respectively. Therefore, custom file formats can be supported by overriding these methods in your own class and passing it into the setConfigClass method. The arguments of loadConfig essentially become the initialization arguments of the class. One of 
the simplest implemented is the DefaultConfig class which simply sets all DAC parameters to their default values and clears all pixel configuration matrices:

>>> tpx.setConfigClass (DefaultConfig)

>>> tpx.loadConfig()

Another is SophyConfig which loads DAC parameters and pixel matrices from Sophy .spx files

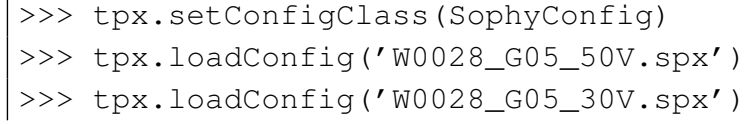

This is also the default configuration class set on TimepixDevice initialization.

\subsection{Data processing pipeline}

Embedded within each TimepixDevice object is a parallel data-pipeline that performs the data-processing for the respective Timepix device. The output of the pipeline, and by extension the callbacks and poll method of Pymepix, is determined by assigning an appropriate AcquisitionPipeline class through the setupAcquisition method.

| >> tpx.setupAcquisition (PixelPipeline)

The AcquisitionPipeline can be thought of as a recipe on how to build the data-pipeline using BasePipelineobjects, which handle the actual processing. Each BasePipelineobject is in-fact a Python multiprocessing.Process with a process method that can generate or perform work on any piece of data in the form of messages. Therefore, the AcquisitionPipeline builds and connects up all the necessary BasePipelineObject in the correct order, spawns their processes and manages the flow of data through the pipeline. The pipeline is built each time the start method is called in Pymepix and is destroyed by stop. Some predefined AcquisitionPipelines include the PixelPipeline that simply reads from UDP and decodes pixels and triggers or the CentroidPipeline that adds a centroiding stage. The currently set AcquisitionPipeline is accessed through the acquisition property in TimepixDevice. For example, in event mode, PixelPipeline can enable pixel and trigger correlation through its enableEvents property:

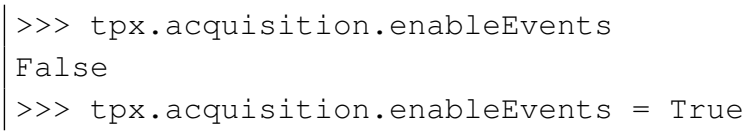

These pipelines can be augmented further by attaching your own BasePipelineObject. The following code snippet demonstrates a simple custom concrete BasePipelineobject:

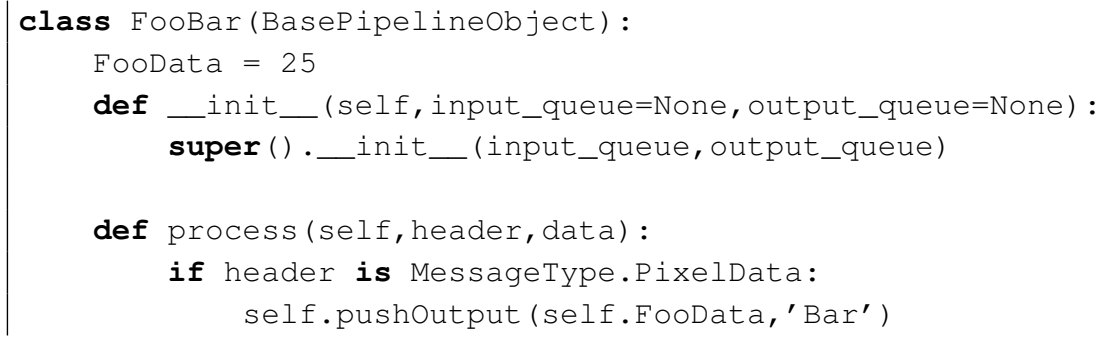

The input_queue and output_queue are the input and output multiprocessing.Queues, respectively, that handle movement of data. A user only needs to know that they are required for the class to function, but do not have to interact with it. In the code above, we see that the process method accepts a message header 
and message data as parameters, similar to callbacks in Listing 2.2, with the movement of the result to the next stage carried out by pushoutput using a unique message type integer identifier FooData. Attaching it to the pipeline can be done using the addStage method:

| >> tpx.acquisition.addStage (10, FooBar)

The first argument is the stage number and define what order the messages flow through each pipeline object in the pipeline. Lower numbers are earlier in the pipeline. Starting an acquisition now shows the new message from our class:

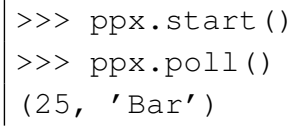

Some of the main specific BasePipelineob ject classes defined in PymePix are briefly described in the following:

UdpSampler This pipeline object creates a UDP socket connection to Timepix 3 and immediately begins collecting UDP packets. UDP packets are converted into arrays and timestamped before being sent off to the next stage with the MessageType. RawData identifier.

PacketProcessor PacketProcessor processes messages with the header MessageType. RawData. It has two modes of operation, pixel mode and event mode. In pixel mode, each 64-bit pixel packet is decoded into their representative column, row, time of arrival (ToA), time over threshold (ToT) and SPIDR timestamp. The time of arrival timestamp range is first extended from $407 \mu$ s to $26.8 \mathrm{~s}$ using the SPIDR timestamp. Using the attached 48-bit global timestamp from the UdpSampler message, the range of unambiguity is further extended to 81.4 days. If fast time of arrival is enabled, then this is applied to the timestamp improving its resolution from $25 \mathrm{~ns}$ to $1.56 \mathrm{~ns}$. Finally, the timestamp is converted from integer nanoseconds into double precision seconds. The results are pushed into the output queue with the MessageType.PixelData Any triggers encountered are also decoded and their timestamp extended in the same fashion as the pixel. The output has the MessageType.TriggerData identifier.

Event mode follows the same procedure as pixel mode but here the trigger and pixel data are cached. When a certain number of triggers have been collected, the cached triggers are then used as bins to assign the pixel ToA to their appropriate triggers using the numpy . digitize method. Each pixel has an assigned trigger number and their ToA are computed as relative to their assigned triggers time. The output has the identifier MessageType.EventData.

Centroiding Centroiding works on MessageType.EventData messages to improve spatial and temporal resolution by centroiding. Since we are working with three-dimensional data $(x, y, \mathrm{ToF})$, flood fill algorithms are unsuitable as they risk destroying events that occur later within the same pixel. Instead the DBSCAN [13] algorithm within the scikit-learn [14] package was chosen as most other clustering algorithms either require a priori knowledge of the number of clusters or were not fast enough. For our dataset, the euclidean metric is used, i. e., the $\epsilon$ term represents the maximum distance radius that points should be considered as part of a cluster. Since the event ToA operates on a different scale, it is necessary to introduce an extra $\epsilon_{f}$ term that is the maximum time distance between pixels. The event ToA are scaled as $t_{f}^{\prime}=\epsilon / \epsilon_{f} \cdot t_{f}$, where $t_{f}$ is the original event time and $t_{f}^{\prime}$ is the scaled event ToA. The default values used are empirically chosen as $\epsilon=3 \mathrm{~ns}$ and $\epsilon_{f}=500 \mathrm{~ns}$ as these values give good results in our analysis. Once the clusters are established, the centroided $x$, $y$, and event time are computed as a weighted mean of all points with respect to their ToT. The centroids final ToT is the maximum ToT in the cluster. The output is packaged under the MessageType. CentroidData. 


\subsection{Timewalk Correction}

Timewalk is an often discussed effect in the literature involving Timepix 3 and improves the temporal resolution by calibrating out a dependency of the ToA on the ToT. The standard operating procedure [15] is to first calibrate the pixels. This calibration is well known, e. g., already from Timepix detectors [16]. To assist with this the pymepix.util module provides the helper function generate_timewalk_lookup that generates a timewalk lookup array for a given ToA/ToF region and ToA/ToF and ToT array. Time arrays from the MessageType.PixelData, MessageType.EventData, and MessageType.CentroidData can be used. The function works similarly to the methods in literature $[15,17]$ by first assuming a "true" arrival time computed from averaging the ToA within a narrow slice of the highest ToTs within the region. All ToAs are converted into time difference from the "true" ToA, are sampled for each ToT, and fit to a Gaussian whose expectation value $\mu$ is the timewalk value. Since the ToT is a 10 bit value, there are only 1024 possible values it can take. The lookup table is generated for all possible ToT values. The index $i$ for a given ToT $t$ in nanosecond can be easily computed as

$$
i=\frac{t}{25}-1
$$

The resulting array can be stored or used by the user to correct pixel or event data after acquisition.

\section{Enduser utility programs}

Included with pymepix are two programs, pymepix-acq and pymepixviewer, that allow for the immediate connection and acquisition of Timepix 3 "out of the box".

\subsection{Command-line data acquisition with pymepix-acq}

pymepix-acq is a command line code using the PymePix library to acquire from a single Timepix device. Its help output is:

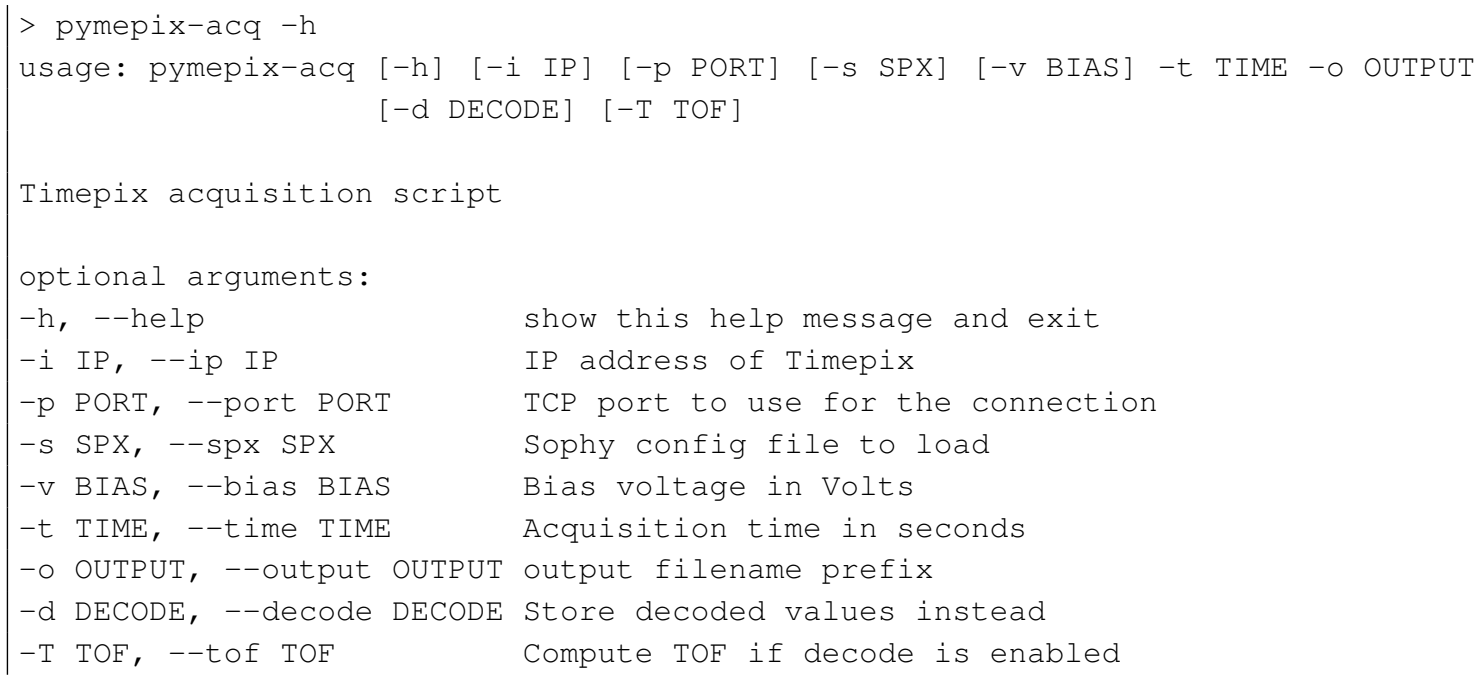

The application can be used from the command-line by specifying an output filename and time in seconds:

|pymepix-acq --time 10 --output my_file

by default only UDP packets are stored with the . raw file extension in raw byte format which includes the 48-bit Timepix 3 clock packets. To store decoded pixel values the $-d$ switch can be used: 
pymepix-acq -d --time 10 --output my_file

this stores the pixel data format specified in Table 1 through successive numpy . save statements into a single file with the .toa file extension. Loading the data in an user program is achieved by repeatedly executing four numpy . load, for $x, y$, global ToA, and ToT, respectively, until an end of file exception occurs. Adding the additional $-T$ switch will activate the event selection mode and will store ToF data in a file with the . tof file extension. This is similar to the previous format, but an additional numpy load is required for the trigger number array.

\subsection{Graphical user interface pymepixviewer}

A separate online viewer is included, built using the PymeP ix, pyqtgraph and PyQt5 libraries. pymepixviewer acts as both a demonstration of the PymePix library and as a simple yet fully-fledged acquisition program. pymepixviewer is geared towards ion imaging, in particularVMI experiments [18, 19], but it is still capable of general use. The viewer can acquire and store all formats described in subsection 3.1, but with the addition of the centroided data given in Table $1 \mathrm{as} . \mathrm{bl}$ ob files. There are three modes available in the viewer: 'ToA mode', 'ToF mode', and 'centroiding mode'. In ToA mode, the viewer can display an integrated image over a specified time range. In ToF mode, a time of flight spectrum is also displayed in Figure 2. The ToF spectrum can be used to define a number of time-ranges, so-called "gates", and labelled by the user to generate new plots in the viewer that display and update data from the selected gates as pixel images. This can be seen in Figure 3 where the top plot is the original "image" from the Timepix 3 detector and the bottom is a gated plot created by the user. Centroiding mode provides the same features as ToF mode, but with the addition of using the higher spatial and
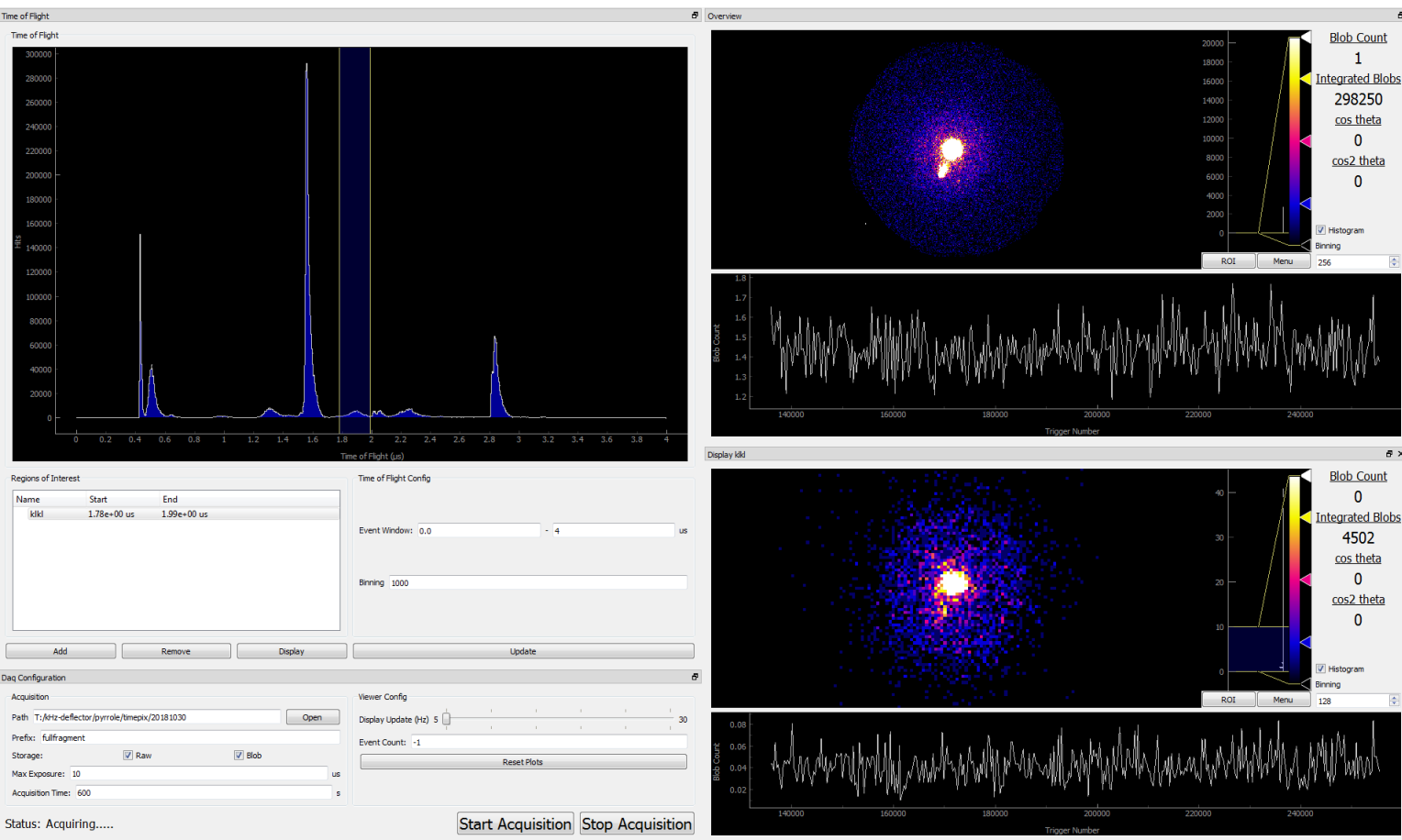

Figure 1. A screenshot of the pymepixviewer GUI in operation. Shown are a live ToF spectrum in the upper left including a ToF gate as blue-shaded area, centroiding, ToF gating, and histograms over the full data in the upper right and the data from the gate in the ToF spectrum. 


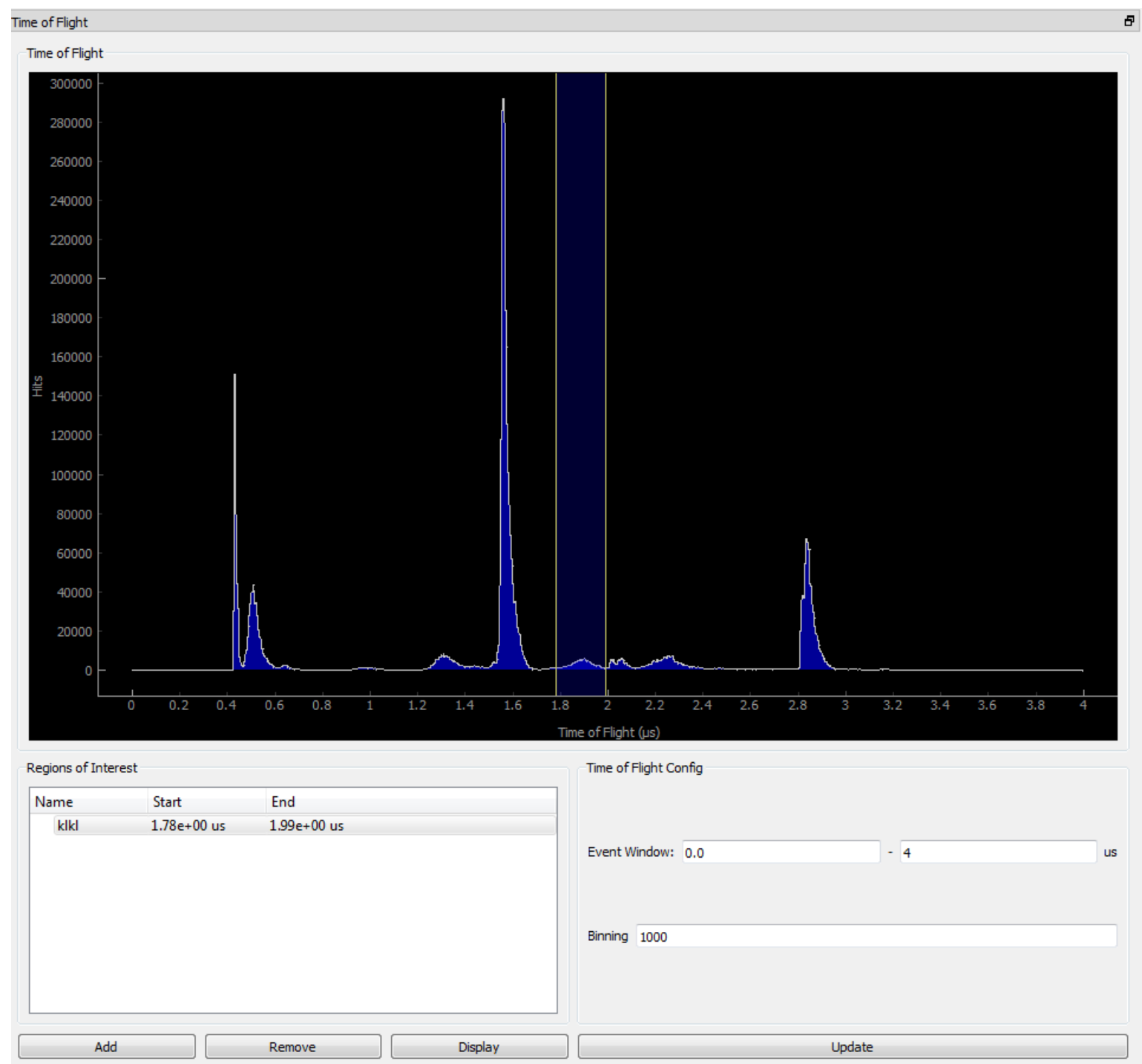

Figure 2. A section of the pymepixviewer GUI displaying a time of flight spectrum and gate creation and selection. The shaded region corresponds the to gate defined in the table

temporal resolution of the reduced centroided data in both the ToF spectrum and the pixel plots. An additional plot can be enabled underneath each pixel plot that shows the number of clusters, so-called "blobs", recorded at each event/trigger number, which can be used for experimental parameter optimization. All of these modes can be switched between on-the-fly. Figure 4 displays the acquisiton control widget which allows the user to set storage location, what is stored, acquisition time and to begin the acquisition itself.

\section{Conclusion}

Timepix 3 is a high-performance high-throughput time-resolving pixelated detector. Python is growing to become a standard language in scientific applications. Connecting these worlds, PymePix is an open-source python 


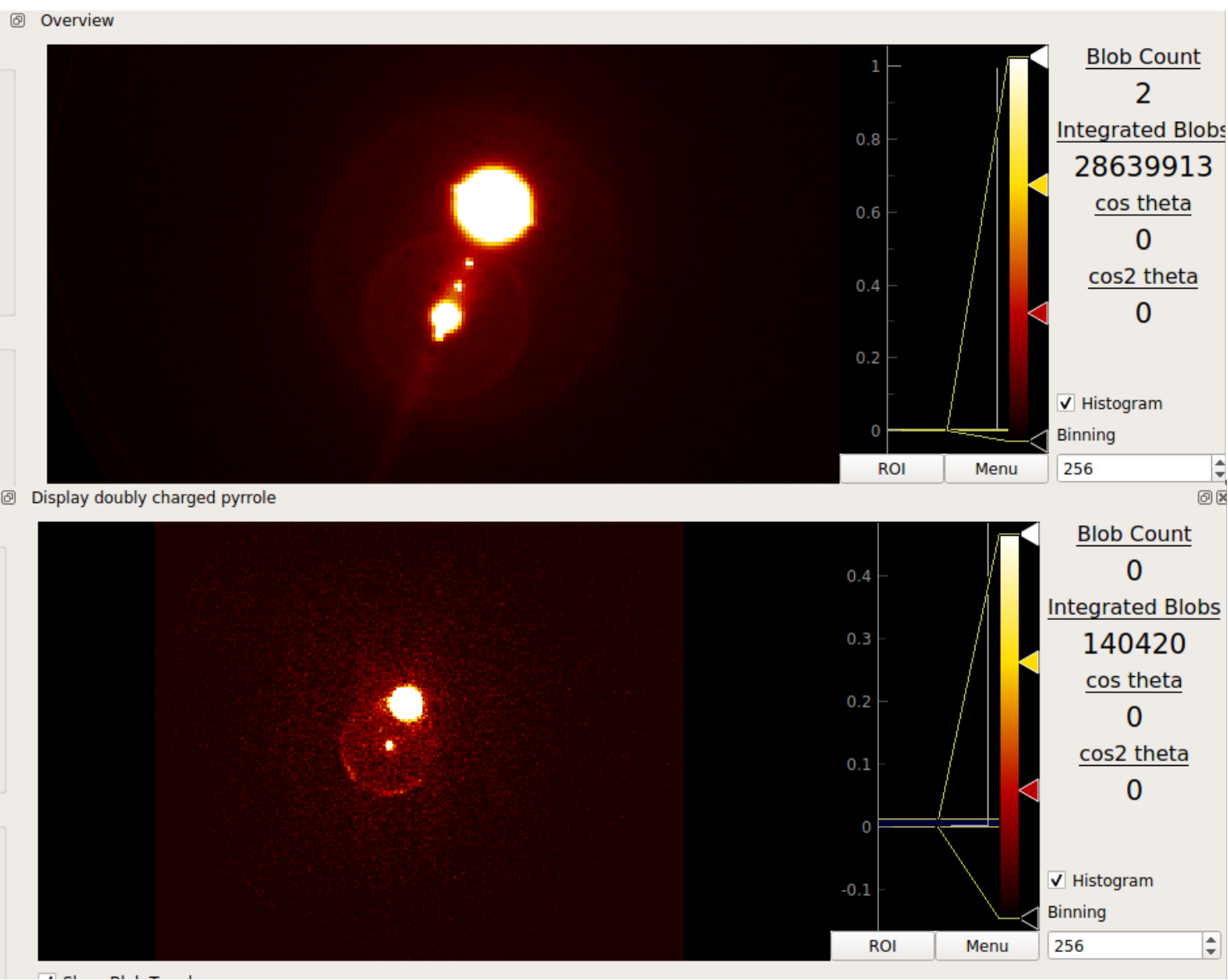

Figure 3. This figure highlights the gating feature of pymepixviewer where the top plot is the full image seen from timepix and the bottom is a "gated" image created by the user.

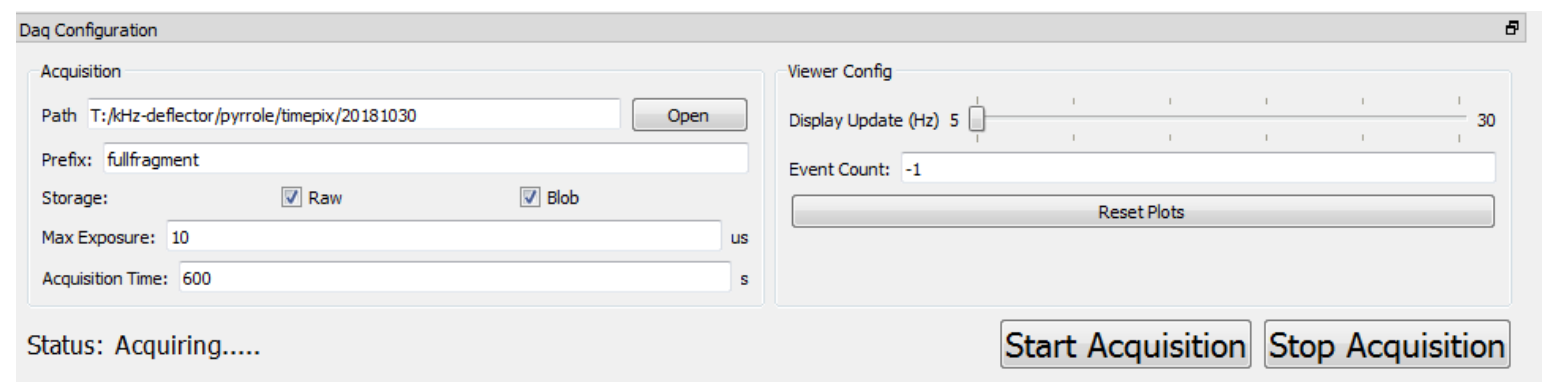

Figure 4. A section of the pymepixviewer GUI highlighting acquisition control and storage settings

library for communicating with and acquiring data from Timepix3, using SPIDR, and simplifies the hardware's control, acquisition, and the analysis of its data. It uses a human-understandable abstraction of the hardware whilst also allowing for fine-grained control. We described an extendible data pipeline to provide a simple way of retrieving and processing the Timepix 3 datastream whilst maintaining performance. Included are programs 
that serve to not only demonstrate the ease of use of PymePix, but to also allow the community to rapidly understand and make use of the powerful Timepix 3 detector.

We point out that in applications such as, for instance, ion or electron imaging a detector system built from Timepix3, SPIDR, and PymePix, introduced here, can easily enable single-shot acquisition of images at very high repetition rate, such as at current or existing free-electron laser facilities [20-22] or high-repetition rate table-top laser systems [23, 24].

\section{Program Availablity}

The library and programs are available under the GPLv3 license from a git repository at https://stash . desy.de/scm/cmipublic/timepix.git and the Python pip repository.

\section{Acknowledgments}

We gratefully acknowledge helpful discussions with Heinz Graafsma regarding Timepix 3 capabilities and use. Igor Rubinskiy had originally started work with Timepix 3 in our group and Ruth Livingstone and Milija Sarajlic had contributed to early experiments. Some low-level codes for the programming of SPIDR were obtained from the $\mathrm{C}++$ code developed by Henk Boterenbrood and colleagues at Nikhef and Amsterdam Scientific Instruments and we gratefully appreciate the sharing of these with us.

This work has been supported by the Clusters of Excellence "Center for Ultrafast Imaging" (CUI, EXC 1074, ID 194651731) and "Advanced Imaging of Matter" (AIM, EXC 2056, ID 390715994) of the Deutsche Forschungsgemeinschaft (DFG) and by the European Research Council under the European Union's Seventh Framework Program (FP7/2007-2013) through the Consolidator Grant COMOTION (ERC-Küpper-614507) and the European Union's Horizon 2020 research and innovation program under the Marie Skłodowska-Curie Grant Agreement 641789 "Molecular Electron Dynamics investigated by Intense Fields and Attosecond Pulses" (MEDEA).

\section{References}

[1] T. Poikela, J. Plosila, T. Westerlund, M. Campbell, M. De Gaspari, X. Llopart, V. Gromov, R. Kluit, M. van Beuzekom, F. Zappon, V. Zivkovicd, C. Brezinae, K. Desche, Y. Fue and A. Kruth, Timepix3: a 65K channel hybrid pixel readout chip with simultaneous ToA/ToT and sparse readout, J. Instrum. 9 (2014) C05013.

[2] X. Llopart, R. Ballabriga, M. Campbell, L. Tlustos and W. Wong, Timepix, a 65k programmable pixel readout chip for arrival time, energy and/or photon counting measurements, Nucl. Instrum. Meth. A 581 (2007) 485-494.

[3] E. Frojdh, M. Campbell, M. D. Gaspari, S. Kulis, X. Llopart, T. Poikela and L. Tlustos, Timepix3: first measurements and characterization of a hybrid-pixel detector working in event driven mode, J. Instrum. 10 (2015) C01039.

[4] J. Visser, M. van Beuzekom, H. Boterenbrood, B. van der Heijden, J. Muñoz, S. Kulis, B. Munneke and F. Schreuder, SPIDR: a read-out system for medipix3 \& timepix3, J. Instrum. 10 (2015) C12028.

[5] B. van der Heijden, J. Visser, M. van Beuzekom, H. Boterenbrood, S. Kulis, B. Munneke and F. Schreuder, SPIDR, $a$ general-purpose readout system for pixel ASICs, J. Instrum. 12 (2017) C02040.

[6] G. Bradski, The OpenCV library, Dr. Dobb's Journal of Software Tools (2000) .

[7] E. Jones, T. Oliphant, P. Peterson et al., SciPy: Open source scientific tools for Python, 2001- [accessed 21. April 2019].

[8] J. D. Hunter, Matplotlib: a 2D graphics environment, Computing in Science \& Engineering 9 (2007) 90-95. 
[9] F. Pérez and B. E. Granger, IPython: A system for interactive scientific computing, Computing in Science \& Engineering 9 (2007) 21-29.

[10] Amsterdam Scientific Instruments, 2019.

[11] Y.-P. Chang, D. A. Horke, S. Trippel and J. Küpper, Spatially-controlled complex molecules and their applications, Int. Rev. Phys. Chem. 34 (2015) 557-590, [1505.05632].

[12] T. Kierspel, C. Bomme, M. Di Fraia, J. Wiese, D. Anielski, S. Bari, R. Boll, B. Erk, J. S. Kienitz, N. L. M. Müller, D. Rolles, J. Viefhaus, S. Trippel and J. Küpper, Photophysics of indole upon $x$-ray absorption, Phys. Chem. Chem. Phys. 20 (2018) 20205, [1802.02964].

[13] M. Ester, H.-P. Kriegel, J. Sander and X. Xu, A density-based algorithm for discovering clusters a density-based algorithm for discovering clusters in large spatial databases with noise, in Proceedings of the Second International Conference on Knowledge Discovery and Data Mining (KDD’96), pp. 226-231, Association for the Advancement of Artificial Intelligence (AAAI), 1996.

[14] F. Pedregosa, G. Varoquaux, A. Gramfort, V. Michel, B. Thirion, O. Grisel, M. Blondel, P. Prettenhofer, R. Weiss, V. Dubourg, J. Vanderplas, A. Passos, D. Cournapeau, M. Brucher, M. Perrot and E. Duchesnay, Scikit-learn: Machine learning in Python, J. Mach. Learn. Res. 12 (2011) 2825-2830.

[15] D. Turecek, J. Jakubek and P. Soukup, USB 3.0 readout and time-walk correction method for Timepix3 detector, J. Instrum. 11 (2016) C12065-C12065.

[16] J. Jakubek, Precise energy calibration of pixel detector working in time-over-threshold mode, Nucl. Instrum. Meth. A 633 (2011) S262-S266.

[17] A. Zhao, M. van Beuzekom, B. Bouwens, D. Byelov, I. Chakaberia, C. Cheng, E. Maddox, A. Nomerotski, P. Svihra, J. Visser, V. Vrba and T. Weinacht, Coincidence velocity map imaging using Tpx3Cam, a time stamping optical camera with 1.5 ns timing resolution, Rev. Sci. Instrum. 88 (2017) 113104, [1707. 06253].

[18] A. T. J. B. Eppink and D. H. Parker, Velocity map imaging of ions and electrons using electrostatic lenses: Application in photoelectron and photofragment ion imaging of molecular oxygen, Rev. Sci. Instrum. 68 (1997) 3477-3484.

[19] S. Trippel, T. Mullins, N. L. M. Müller, J. S. Kienitz, K. Długołęcki and J. Küpper, Strongly aligned and oriented molecular samples at a kHz repetition rate, Mol. Phys. 111 (2013) 1738, [1301 . 1826].

[20] J. Feldhaus, FLASH - the first soft x-ray free electron laser (FEL) user facility, J. Phys. B 43 (2010) 194002.

[21] R. Abela, K. Witte, A. Schwarz, H. Redlin, H. J. Eckoldt, M. Hartrott, K. Floettmann, M. Fajardo, V. Katalev, T. Schilcher, K. Tiedtke, J. Baehr, W. Singer, E. Sombrowski, S. Casalbuoni, J. Hajdu, J. Schneider, G. Dipirro, J. W. Kim, R. Brinkmann, B. Racky, J. Chen, A. Nienhaus, H. Chapman, H. Lierl, E. Gadwinkel, C. Gerth, I. Robinson, S. Choroba, M. Maslov, R. Wenndorff, C. Pagani, G. Tallents, H. J. Grabosch, E. Jaeschke, R. Paparella, P. Seller, P. Piot, I. Tsakov, D. Kraemer, A. Winter, M. Sachwitz, A. Cianchi, H. Weddig, W. Sandner, C. Schroer, T. Nunez, P. Castro, O. Napoly, Q. Kong, S. Simrock, U. Gensch, H. Thom, S. Bratos, C. Altucci, B. Dobson, C. Masciovecchio, H. Quack, R. Kienberger, M. Wendt, M. Chergui, O. Grimm, A. Plech, F. Obier, B. Schmidt, V. Sytchev, J. Rossbach, O. Kozlov, M. Kuhlmann, F. Senf, A. Wolf, J. Vogel, J. Prenting, F. Stephan, E. Syresin, D. Sertore, W. Decking, N. Baboi, E. Vogel, L. Juha, A. Brandt, E. Collet, Y. Filipov, I. Vartaniants, J. Marangos, N. Golubeva, R. Smith, S. Techert, A. Fateev, M. Wulff, L. Haenisch, M. Huening, V. Honkimäki, R. Treusch, H. Ihee, S. Prat, B. Petrosyan, E. Prat, M. Tolan et al., XFEL: The European X-Ray Free-Electron Laser-Technical Design Report. DESY, 2006.

[22] New science opportunities enabled by LCLS-II x-ray lasers, Tech. Rep. SLAC-R-1053, SLAC National Accelerator Laboratory, June, 2015.

[23] J. Rothhardt, S. Hädrich, Y. Shamir, M. Tschnernajew, R. Klas, A. Hoffmann, G. K. Tadesse, A. Klenke, T. Gottschall, T. Eidam, J. Limpert, A. Tünnermann, R. Boll, C. Bomme, H. Dachraoui, B. Erk, M. Di Fraia, D. A. Horke, T. Kierspel, T. Mullins, A. Przystawik, E. Savelyev, J. Wiese, T. Laarmann, J. Küpper and D. Rolles, 
High-repetition-rate and high-photon-flux $70 \mathrm{eV}$ high-harmonic source for coincidence ion imaging of gas-phase molecules, Opt. Exp. 24 (2016) 18133-18147.

[24] F. J. Furch, T. Witting, A. Giree, C. Luan, F. Schell, G. Arisholm, C. P. Schulz and M. J. J. Vrakking, CEP-stable few-cycle pulses with more than $190 \mu \mathrm{J}$ of energy at $100 \mathrm{kHz}$ from a noncollinear optical parametric amplifier, Opt. Lett. 42 (2017) 2495-2498. 